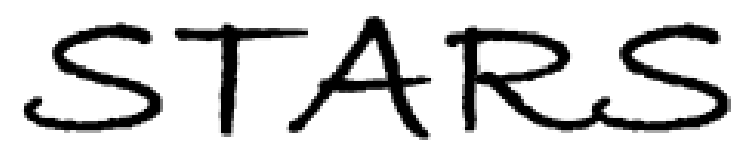

University of Central Florida

STARS

$1-1-1997$

\title{
Practical considerations on color image enhancement using homomorphic filtering
}

Liviu I. Voicu

University of Central Florida

Harley R. Myler

University of Central Florida

Arthur R. Weeks

University of Central Florida

Find similar works at: https://stars.library.ucf.edu/facultybib1990

University of Central Florida Libraries http://library.ucf.edu

This Article is brought to you for free and open access by the Faculty Bibliography at STARS. It has been accepted for inclusion in Faculty Bibliography 1990s by an authorized administrator of STARS. For more information, please contact STARS@ucf.edu.

\section{Recommended Citation}

Voicu, Liviu I.; Myler, Harley R.; and Weeks, Arthur R., "Practical considerations on color image enhancement using homomorphic filtering" (1997). Faculty Bibliography 1990s. 2124.

https://stars.library.ucf.edu/facultybib1990/2124

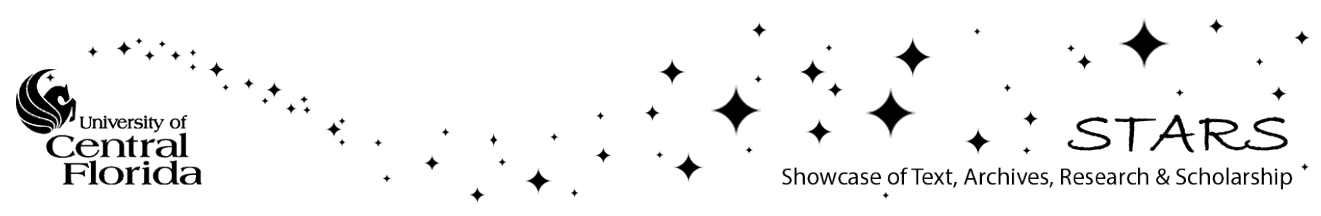




\title{
Practical considerations on color image enhancement using homomorphic filtering
}

\author{
Liviu I. Voicu \\ Harley R. Myler \\ Arthur R. Weeks \\ University of Central Florida \\ Department of Electrical and Computer Engineering \\ Orlando, Florida 32816-2450 \\ E-mail: hrm@engr.ucf.edu
}

\begin{abstract}
We present a study concerning the practical possibilities of using the homomorphic filtering for color image enhancement. Two of the most popular color models, RGB and C-Y (color difference), are employed and the results are comparatively discussed. The homomorphic filtering has proven to be a viable tool for both color models considered. (c) 1997 SPIE and IS\&T.
\end{abstract}

\section{Introduction}

According to a classical model for intensity images, we can consider an image as the result of the reflection of an illumination source over an object or scene. This model is expressed in terms of intensity spatial distributions, as follows ${ }^{1,2}$ :

$f(x, y)=i(x, y) r(x, y)$,

where $f(x, y)$ represents the image intensity function, $i(x, y)$ represents the intensity of the illumination and $r(x, y)$ describes the spatial distribution of the reflectance of the object or scene.

Typically, the illumination component is characterized by smooth variations within the input plane and its spectrum will be dominated by low spatial frequencies. ${ }^{1,2}$ On the other hand, the spectrum of the reflectance component will be typically distributed about the entire frequency domain. ${ }^{1,2}$ By separating the two components, attenuating the low spatial frequencies and amplifying the high spatial frequencies, the undesired contributions within the image that are due to light source nonuniformities can be reduced and the object features can be emphasized. The logarithmic function can convert Eq. (1) into a linear expression [Eq. (2)], containing two additive components:

$\log [f(x, y)]=\log [i(x, y)]+\log [r(x, y)]$.

Paper 96-019 received Apr. 22, 1996; revised manuscript received July 29, 1996; accepted for publication July 29, 1996.

$1017-9909 / 97 / \$ 10.00$ @ 1997 SPIE and IS\&T.
In mathematical terms, the logarithm defines a homomorphism that maps the image from the multiplicative group of the real positive numbers into the additive group of the real numbers, where a linear filtering process becomes applicable. After taking the Fourier transform of Eq. (2), a linear filter is applied to amplify high frequencies and attenuate low frequencies. The next step is to retrieve the enhanced image by taking the inverse Fourier transform and exponentiating the result. Under the specifications above, one obtains both a dynamic range compression of the graylevel scale and a contrast enhancement. The whole process is completely described by the diagram in Fig. 1 and is known as a homomorphic filter.

The applications of homomorphic filtering are numerous. By equalizing the illumination variations onto the image, one can retrieve details lost in dark regions. The homomorphic filter has also been applied to audio signal enhancement and echoed signal restoration. ${ }^{3}$ In image processing, the possibility of optical implementation for the homomorphic filter makes it a powerful alternative to other enhancement techniques, such as histogram equalization. ${ }^{4}$ An approach to applying homomorphic filtering in color image processing has been also reported ${ }^{5}$ as an argument for a human color vision model based on the logarithmic response of the cone receptors of the retina to the stimulus intensity.

Our goal was to study the possibility of using homomorphic filtering in color image enhancement from a practical point of view. There exist several models for color images ${ }^{2}$ and some of them separate the intensity component of pixels from the color components. The homomorphic filtering of the intensity component seems to be a natural issue due to the similarity with the classical approach. However, intricate problems may occur due to the fact that color parameters cannot be totally separated and they carry some intensity information by themselves. Moreover, the intensity component is defined differently from model to model and its physical meaning is affected accordingly. On the other hand, for the tristimulus-based models, which do not separate the achromatic and chromatic information, Eq. (1) 


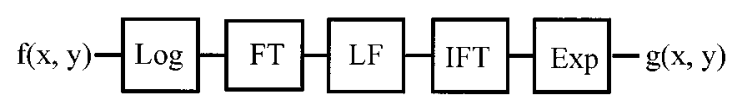

Fig. 1 Process of homomorphic filtering for grayscale images: Log, logarithm; FT, Fourier transform; LF, linear filter; IFT, inverse Fourier transform; Exp, exponential; $f(x, y)$, initial image intensity function; $g(x, y)$, processed image intensity function.

does not apply. Therefore, choosing the most appropriate model for implementing the homomorphic filtering is not an easy problem.

In this paper, we study the implementation of the homomorphic filtering in two of the most popular color models, one from each category, and have comparatively discussed the results.

\section{Homomorphic Filtering in RGB Color Space}

The RGB color model is one of the most common color models used for computer image processing and presents the advantage of simplicity. Moreover, the tristimulusbased models such as RGB are linear with respect to additive mixtures and scaling. ${ }^{6}$ The RGB color space can be geometrically represented by the positive quadrant of a 3-D, orthogonal space, with R, G and B-tristimulus coefficients as coordinates. Typically, each coefficient is represented by a single byte, therefore, the size of the RGB color space is limited to a finite cube, as shown in Fig. 2.

It is important at this point to note that tristimulus values of surfaces can only be considered relatively to the illumination source. When no specification is made, however, it is assumed that the tristimulus values of a given surface are those tristimulus values obtained by exposing that surface to white illumination of intensity one.

Color physics states that light emerging from a surface is described by tristimulus coordinates given by the following equation ${ }^{6}$ :

$T=\int_{\lambda_{1}}^{\lambda_{2}} P_{\lambda} \bar{t}(\lambda) \mathrm{d} \lambda$,

where $T$ represents the tristimulus coefficient (R, G, or B), $\left[\lambda_{1}, \lambda_{2}\right]$ is the wavelength interval for the visible spectrum, $P_{\lambda}$ denotes the spectral radiant power of the illumination source, and $\bar{t}(\lambda)$ is the color-matching coefficient for the

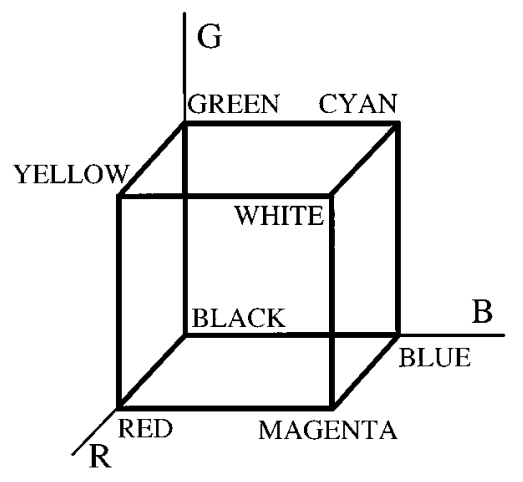

Fig. 2 RGB color cube. corresponding tristimulus. The model requires complete knowledge of the spectral properties of both the source and the object surface and, hence, is difficult to use in practice. Moreover, the metameric sources (sources having the same tristimulus coefficients and therefore the same color appearance, but different spectral power distributions) may produce different colors when illuminating nonwhite surfaces. Surfaces also exist that have the same color appearance under white illumination (and thus, identical tristimulus values), but a different appearance when the illuminant is changed. ${ }^{7}$ The conclusion is that one cannot model exactly the result of a reflection of a colored beam over a certain colored object using only the tristimulus coefficients. However, the following approximation can be used $^{7}$ :

$T_{\text {image }}(x, y) \approx T_{\text {illumination }}(x, y) T_{\text {reflection }}(x, y)$,

where $T$ is the tristimulus coefficient $(\mathrm{R}, \mathrm{G}$, or B). Different studies have shown that this approximation works well in

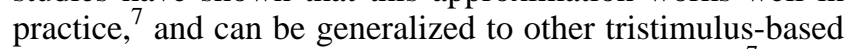
models, such as the model using XYZ primaries. ${ }^{7}$ Each component in Eq. (4) suggests that a homomorphic filtering process as described in Fig. 1 for intensity images can be individually applied to each of the RGB color components. Since the ratios between the R, G and B components of the image may be altered and, hence, the color hue of the pixels may be modified, the process is likely to produce color artifacts. Our results have shown that these color artifacts do not drastically affect the color appearance of the homomorphic filtered image as compared to the perceived improvement due to an increase in contrast dynamic range. However, the process is more suited to applications where the graphic qualities of the image are not a primary issue, such as surveillance, target recognition or image restoration.

\section{Homomorphic Filtering in C-Y (Color Difference) Color Space}

It is generally accepted that human vision can be described by a three-channel model, one channel for the intensity information and two channels for chromatic information. Studies concerning homomorphic filtering using this type of color model have been reported in the literature. ${ }^{5} \mathrm{~A}$ homomorphic filtering was performed on the two types of channels separately, and the results of the possible combinations were studied. However, by filtering the chromatic components independently, different types of color artifacts may appear, as well as undesired saturation and hue biases. Furthermore, the back-mapping into the RGB space of the result may also produce unrealizable colors. ${ }^{8}$

From the models that decouple the chromatic and achromatic information, we have chosen the C-Y (color difference) model due to some special features it presents. Similar to other models, the intensity component Y (achromatic information component) is defined as a linear combination of $\mathrm{R}, \mathrm{G}$ and $\mathrm{B}$ tristimulus coefficients. However, the weights used in the combination are not equal and take into account the differences between perceived brightness for each component. This feature enables the C-Y model to better represent the natural way of perceiving colors, and the physical meaning of the intensity component is, therefore, closer to that of human perception. Moreover, the C-Y 


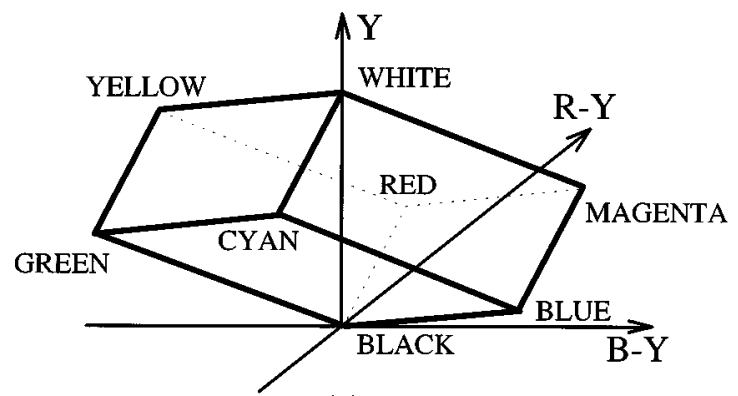

(a)

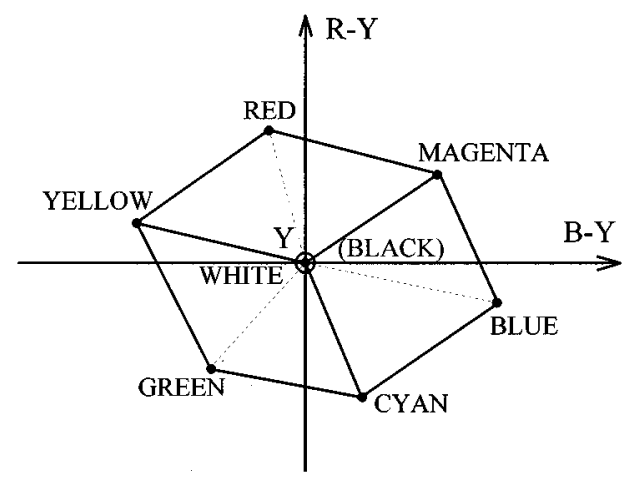

(b)

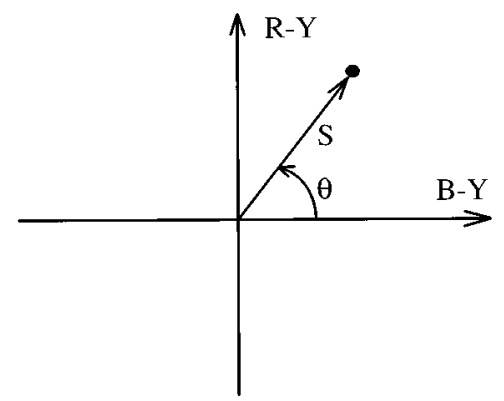

(c)

Fig. 3 Mapping of RGB color cube in the $C-Y$ (color difference) space: (a) 3-D view, (b) projection on (B-Y, R-Y) plane, and (c) hue $\theta$ and saturation $S$, as polar coordinates in the $\mathrm{C}-\mathrm{Y}$ (color difference) space.

model is one of the standards for television broadcasting, ${ }^{9}$ and hence, a large amount of color video hardware is available. This makes real-time implementations of the algorithm feasible.

The C-Y color model coordinates are given by the transformation described in Eq. (5):

$\left[\begin{array}{c}\mathrm{Y} \\ \mathrm{R}-\mathrm{Y} \\ \mathrm{B}-\mathrm{Y}\end{array}\right]=\left[\begin{array}{ccc}0.299 & 0.587 & 0.114 \\ 0.701 & -0.587 & -0.114 \\ -0.299 & -0.587 & 0.886\end{array}\right] \cdot\left[\begin{array}{c}\mathrm{R} \\ \mathrm{G} \\ \mathrm{B}\end{array}\right]$.

This transformation does not preserve distances or angles. Therefore, the mapping of the RGB cube in $\mathrm{C}-\mathrm{Y}$ space, shown in Figs. 3(a) and 3(b), has a nonregular shape. Typically, after performing a certain color image processing technique in the $\mathrm{C}-\mathrm{Y}$ space, it is necessary to map the result back into the RGB space, which is then used by color displays to observe the processed color image. In this case,

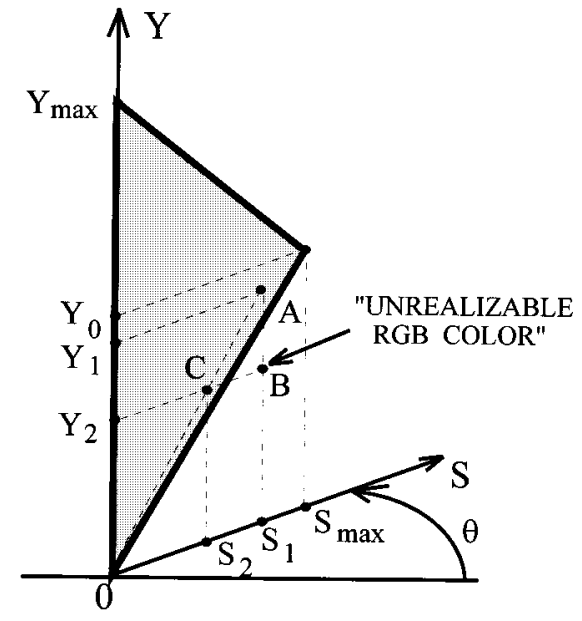

Fig. 4 Graphical representation of the "unrealizable RGB color" problem and the saturation adjustment procedure: $S$, saturation coordinate; $Y$ intensity coordinate; $\theta$, hue coordinate; $S_{1}$ and $Y_{1}$, initial saturation and intensity, respectively; $S_{2}$ and $Y_{2}$, final intensity and saturation, respectively; $S_{\max }$ and $Y_{0}$, maximum saturation obtainable for a particular hue $\theta$ and the corresponding intensity, respectively; and $Y_{\max }$, maximum intensity.

the resulting colors may lie outside the mapping emphasized in Figs. 3(a) and 3(b) and, therefore, may produce color artifacts called "unrealizable RGB colors."

Since the intensity component Y carries the decoupled achromatic information, the color information can be completely described in (B-Y, R-Y) plane. By using polar coordinates, as shown in Fig. 3(c), one can define two parameters with physical meaning, the saturation component $\mathrm{S}$ and hue component $\theta$ as follows:

$S=\left[(\mathrm{R}-\mathrm{Y})^{2}+(\mathrm{B}-\mathrm{Y})^{2}\right]^{1 / 2}$,

$\theta=\arctan \left(\frac{\mathrm{R}-\mathrm{Y}}{\mathrm{B}-\mathrm{Y}}\right)$

A radial cross section of the RGB cube mapping presented in Fig. 3(a), corresponding to a constant hue, can help in redefining the unrealizable RGB color problem. The cross section, depicted in Fig. 4, represents the variation domain for the saturation component that guarantees a proper mapping into the RGB cube. Under these circumstances, every color image processing performed in $\mathrm{C}-\mathrm{Y}$ space should be followed by a saturation adjustment to avoid the creation of unrealizable colors when mapped into the RGB cube. Concretely, if a certain color $A$ (Fig. 4), undergoes an intensity modification from $Y_{1}$ to $Y_{2}$ due to a particular color image processing, and if $A$ has a saturation component that is close to the maximum possible for the corresponding intensity and hue components, the resulting color may lie outside the desired variation domain, at point $B$. Therefore, the saturation adjustment process should map the point $B$ back into the desired domain, at point $C$, in Fig. 4(a).

The RGB image can be then obtained through the inverse transformation: 


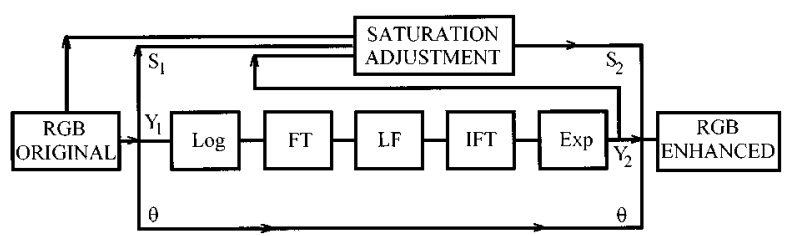

Fig. 5 Process of homomorphic filtering using the $C-Y$ model. The image is converted first into the $\mathrm{C}-\mathrm{Y}$ space, then a homomorphic filtering is applied on the intensity component and, finally, the saturation component is adjusted in order to prevent the creation of "unrealizable RGB colors" and the resulting image is mapped into the RGB space for output: Log, logarithm; FT, Fourier transform; LF, linear filter; IFT, inverse Fourier transform; Exp, exponential; $S_{1}$ and $Y_{1}$ initial saturation and intensity, respectively; $S_{2}$ and $Y_{2}$, final intensity and saturation, respectively; and $\theta$, hue.

$\mathrm{R}=S \sin \theta+\mathrm{Y}$

$\mathrm{B}=S \cos \theta+\mathrm{Y}$

$\mathrm{G}=-0.52(\mathrm{R}-\mathrm{Y})-0.19(\mathrm{~B}-\mathrm{Y})$

The implementation of the homomorphic filtering in $\mathrm{C}-\mathrm{Y}$ space is shown in the block diagram from Fig. 5. First, the intensity component $Y$ undergoes a classical homomorphic filtering process. Then, the result is used to adjust the saturation component of each pixel, according to Fig. 4. The hue component is not affected by the homomorphic filtering in the $\mathrm{C}-\mathrm{Y}$ space.

\section{Linear Filter Implementation}

Except for the linear filter (LF), the operations shown in Fig. 1 do not present any major difficulties. Nevertheless, the optimal function for the filter is strongly dependent on the image features. Early studies concerning the filter shape, ${ }^{1}$ employ two parameters, $\gamma_{L}$ and $\gamma_{H}$ to represent the gain coefficients for low and high frequencies, respectively. A general shape for the filter function is presented in Fig. 6.

In our work, we have used a modified, second order Butterworth filter function, as follows:

$H(\rho)=\gamma_{1}-\gamma_{2} \frac{1}{1+2.415\left(\rho / \rho_{c}\right)^{4}}$,

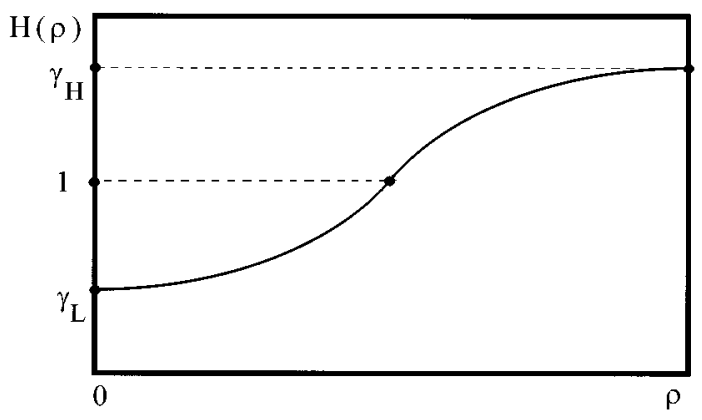

Fig. 6 General shape of the LF function, $H(\rho): \gamma_{H}$, high frequency gain; $\gamma_{L}$, low frequency gain; and $\rho$, spatial frequency coordinate. where $\rho=\left[\left(u-u_{0}\right)^{2}-\left(v-v_{0}\right)^{2}\right]^{1 / 2}$ is the distance between $(u, v)$ and the center of the filter, $u_{0}$ and $v_{0}$ are the center coordinates, and $\rho_{c}$ is the cutoff spatial frequency of the filter. The parameters $\gamma_{1}$ and $\gamma_{2}$ have been added for flexibility. By varying $\gamma_{1}$ and $\gamma_{2}$, one can obtain any value for high and low frequency gains, $\gamma_{H}$ and $\gamma_{L}$, respectively. Indeed, for high frequencies with respect to the cutoff frequency, when $\rho / \rho_{c} \gg 1$, one can derive the relationship: $\gamma_{H} \approx \gamma_{1}$. Also, for low frequencies, when $\rho / \rho_{c} \ll 1$, we have $\gamma_{L} \approx \gamma_{1}-\gamma_{2}$. Additionally, the sharpness of the transition between $\gamma_{L}$ and $\gamma_{H}$ can be controlled by varying the cutoff frequency $\rho_{c}$.

\section{Results}

The first image employed for enhancement is presented in Fig. 7(a). As one can observe, the original image is blurred and most of the background is dark. A linear technique for contrast enhancement will saturate the bright regions while trying to enhance dark regions. The alternative could be a nonlinear technique, which will enhance the light and dark regions differently. In terms of homomorphic filtering, by attenuating low frequencies after taking the logarithm of the image intensity function, one can equalize the illumination component, and details in the dark regions will be retrieved. Also, by amplifying the high frequencies at the same point, we can gain contrast and eliminate the blurring effects.

We have demonstrated that this technique can be applied with good results, both in RGB and C-Y color models. Studies concerning the sensitivity of the image enhancement on the shape of the linear filter, for the particular image in Fig. 7(a), have also been made. The results are presented in Figs. 7(b) and 7(c) for RGB and C-Y, respectively, and they show that a good contrast improvement can be obtained with a smooth filter (Fig. 8) characterized by the following parameters: $\gamma_{L}=0.8, \gamma_{H}=1.8$, and $\rho_{c}=100$. Note that the details have become more visible and the blurring effect has been attenuated. However, the equalization of the illumination was not very effective. We successively applied a second homomorphic filter, on the images shown in Figs. 7(b) and 7(c), with an abrupt shape (Fig. 10). The final images are presented in Figs. 9(a) and 9(b), and one can easily observe the person in the background, which is very hard to distinguish in the original image [Fig. 7(a)] and even after the first processing [Figs. 7(b) and 7(c)]. The linear filter employed in the second process is characterized by the following parameters: $\gamma_{L}=0.4, \gamma_{H}$ $=1.0$, and $\rho_{c}=2$. For a better appearance, the final image has been enhanced in brightness.

As stated in the introductory section, the beneficial effects of the homomorphic filtering process rely on two basic features, the capability of selectively enhancing the contrast on details and of equalizing the illumination component over the image plane. Our study shows that, for the best efficiency, one should separate the two effects and treat them differently. Indeed, one can hardly obtain bothtypes of enhancement using a single linear filter. The most efficient enhancement of a blurred image calls for a smooth filter function with a gain greater than one for the high frequency domain. On the other hand, if an image needs an illumination equalization, the most appropriate filter is a 


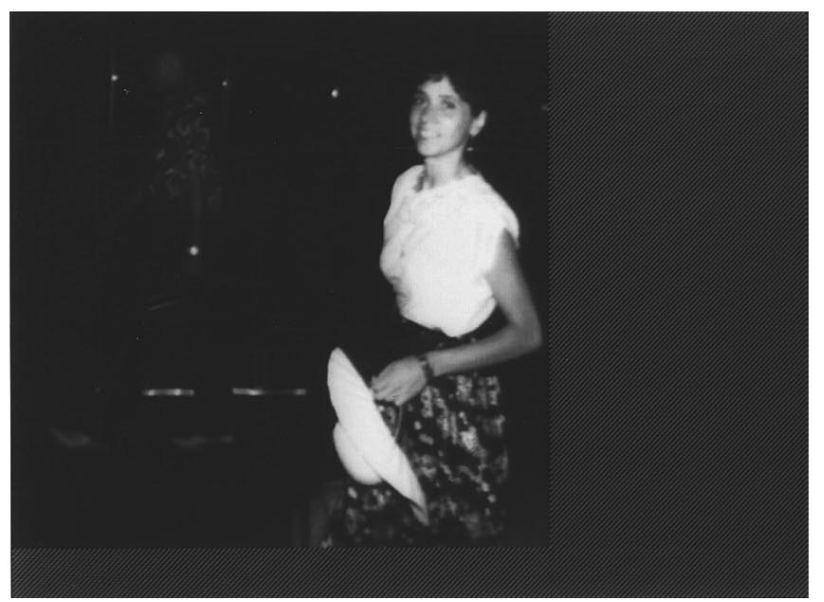

(a)

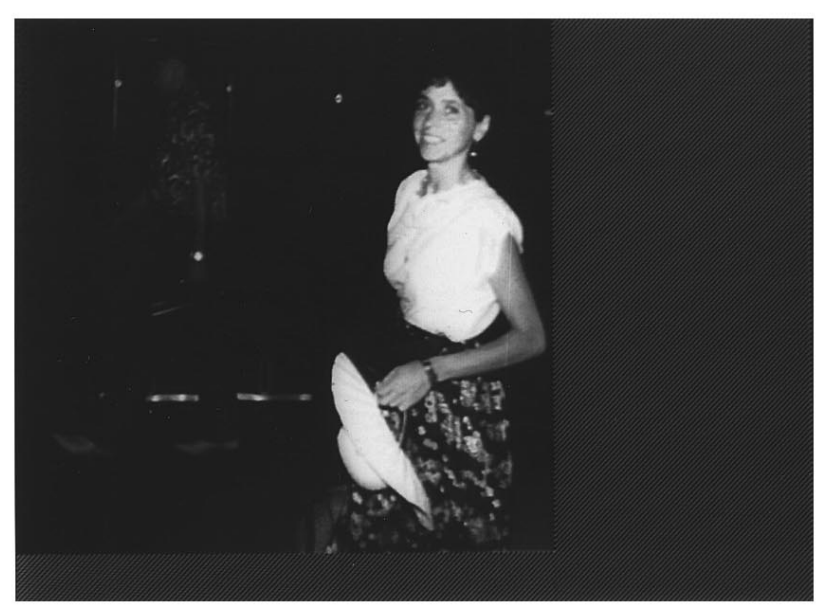

(b)

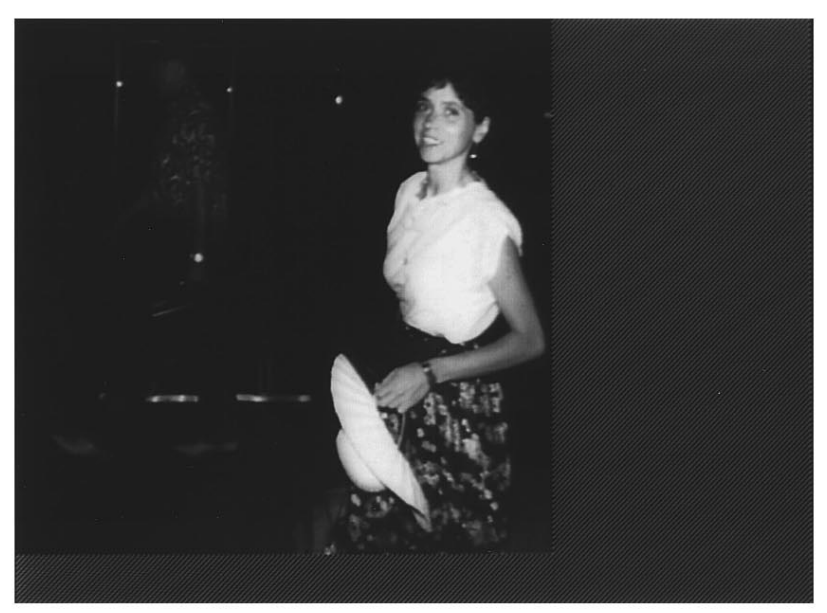

(c)

Fig. 7 (a) Original test image, (b) enhanced using homomorphic filtering in the RGB space, and (c) enhanced using homomorphic filtering in the C-Y space. The enhancements in (b) and (c) were performed with the filtering function presented in Fig. 8.

sharp one that affects only a small interval around the origin.

Finally, it is important to note that the same linear filter has produced the best results in both color models, which

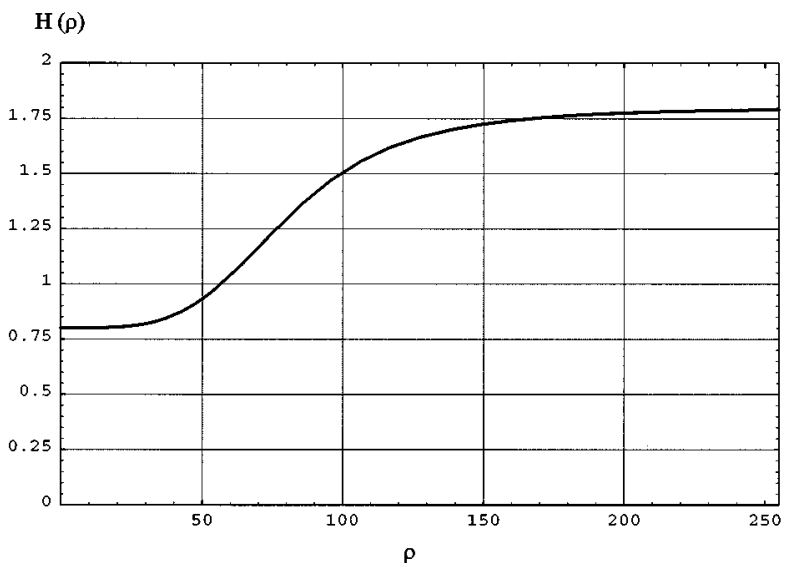

Fig. 8 Shape of the linear filter with $\gamma_{L}=0.8, \gamma_{H}=1.8$, and $\rho_{C}=100$ (cross section).

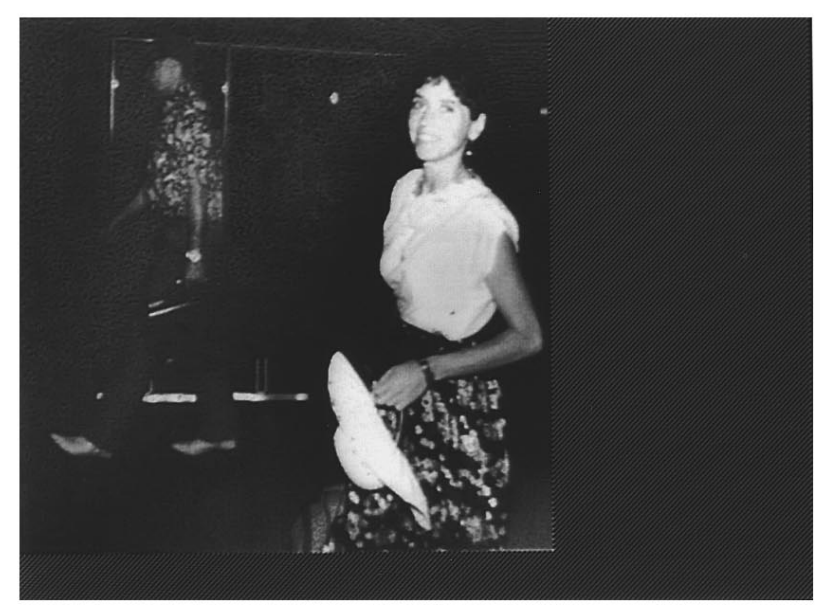

(a)

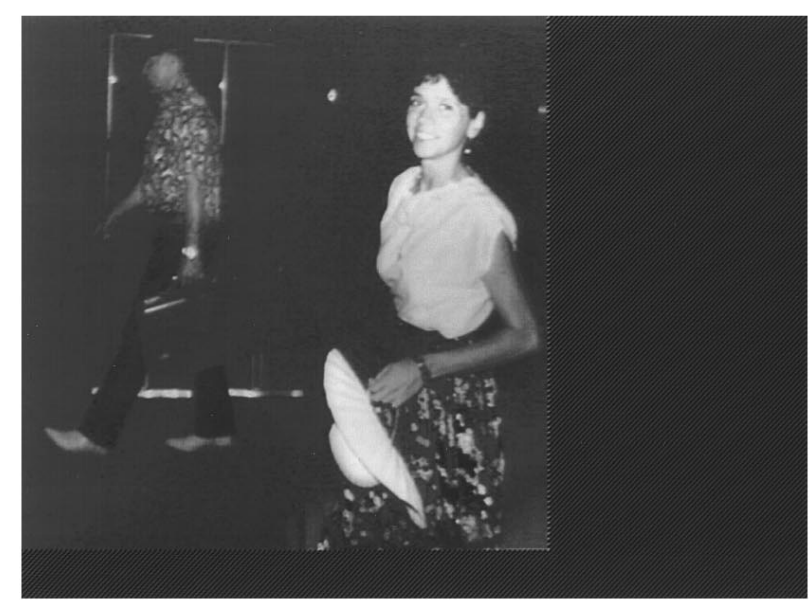

(b)

Fig. 9 Images after the second enhancement: (a) in the RGB space and (b) in the C-Y space. The enhancements in (a) and (b) were performed with the filter function presented in Fig. 10.

proves that the optimal filter function depends only on the spectral features of the image and does not depend on the color appearance or color model chosen.

The homomorphic filter process may produce some in- 
$\mathbf{H}(\rho)$

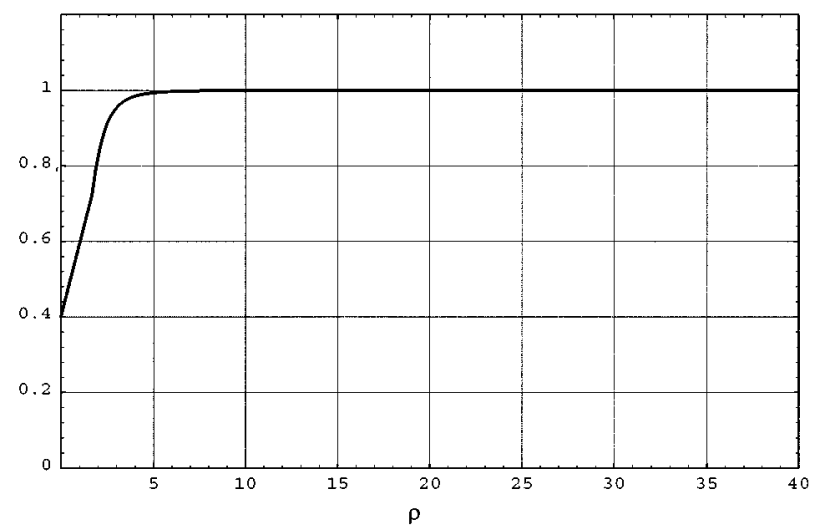

Fig. 10 Shape of the linear filter with $\gamma_{L}=0.4, \gamma_{H}=1.0$, and $\rho_{C}=2$ (cross section).

herent color artifacts, especially when using the RGB model. It is possible that these artifacts will affect the subjective quality of an image and, therefore, homomorphic filtering might not be a solution in applications concerned with image quality in terms of color correctness.

The implementation was performed on a Sun SPARC workstation using the $\mathrm{C}$ programming language. The base of the logarithm employed by the homomorphic process was chosen equal to 2 to obtain a better resolution and for computational reasons. Accordingly, the final operation of the homomorphic filtering process was to raise 2 to the powers given by the pixel graylevels. The original picture was scanned and converted into $512 \times 512 \times 24$ bit raw format.

\section{Conclusions}

We studied, from a practical point of view, the possibility of employing classical homomorphic filtering, typically used in grayscale images, to enhance a color image. Two fundamentally different color models have been employed with good results. In the RGB model, the homomorphic filtering can be performed by considering each color component, separately. On the other hand, in the C-Y (color difference) model, the homomorphic filtering, performed on the intensity component, must be followed by a saturation adjustment to ensure the mapping of the result in RGB space without producing unrealizable RGB colors. We have also studied the effects of different shapes for the linear filter on the quality of enhancement and conclude that a higher quality may be obtained by using distinct filters successively.

\section{Note}

Results of the filtering techniques presented in this paper are available on the World Wide Web at: http://www-ece.engr.ucf.edu/miil/publications.html.

\section{Acknowledgments}

The authors would like to thank the two reviewers for their valuable observations and useful comments.

\section{References}

1. A. V. Oppenheim and R. W. Schaffer, Digital Signal Processing, pp 487-490, Prentice-Hall, Englewood Cliffs, NJ (1975).

2. R. C. Gonzales and P. Wintz, Digital Image Processing, pp. 225-237, Addison-Wesley, Reading, MA (1987).

3. A. V. Oppenheim, R. W. Schaffer, and T. G. Stockham, "Nonlinear filtering of multiplied and convolved signals,' Proc. IEEE 56, 12641286 (1968).

4. A. R. Weeks, H. R. Myler, and J. D. Emery, "Implementation of a hybrid optical homomorphic filter using a liquid crystal television as a spatial light modulator,' Opt. Eng. 31(9), 1986-1989 (1992).

5. O. D. Fagueras, "Digital color image processing within the framework of a human visual model," IEEE Trans. Acoust. Speech Signal Process. ASSP-27(4), 380-393 (1979).

6. G. Wyszecki and W. S. Stiles, Color Science: Concepts and Methods, Quantitative Data and Formulae, 2nd ed., pp. 143, 157-158, John Wiley \& Sons, New York (1982).

7. C. F. Borges, "Trichromatic approximation method for surface illumination," J. Opt. Soc. Am. A 8(8), 1319-1323 (1991).

8. A. R. Weeks, G. E. Hague, and H. R. Myler, "Histogram equalization of 24-bit color images in the color difference (C-Y) color space,' $J$. Electron. Imaging 4(1), 15-22 (1995).

9. A. V. J. Martin, Technical Television, pp. 498-500, Prentice-Hall, Englewood Cliffs, NJ (1962).

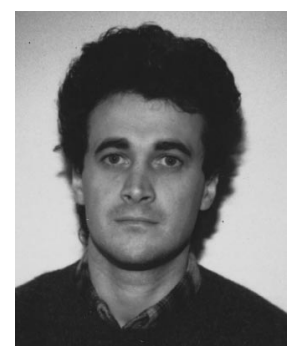

Liviu I. Voicu received his engineer diploma degree in electronics and telecommunications from the University Politehnica of Bucharest, Romania, in 1988. He was a research assistant at the Institute of Atomic Physics in Bucharest from 1990 to 1994. He is currently pursuing a PhD program in electrical engineering at the University of Central Florida in Orlando. His main research interests are color vision and machine intelligence, particularly in learning algorithms with biomemetic properties. Voicu is a member of the IEEE.

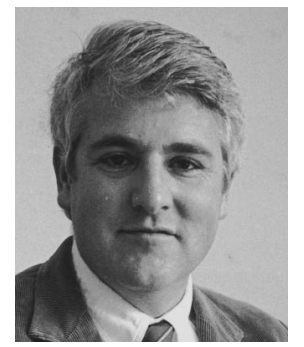

Harley R. Myler is an associate professor of engineering in the Department of Electrical and Computer Engineering at the University of Central Florida in Orlando. He received his BSEE from the Virginia Military Institute in 1975 and MSEE and PhD degrees from New Mexico State University in 1982 and 1985, respectively. Dr. Myler's primary research interest is machine intelligence with emphasis on image understanding and intelligent control. $\mathrm{He}$ is a senior member of the IEEE, a member of SPIE, and a Tau Beta Pi Eminent Engineer.

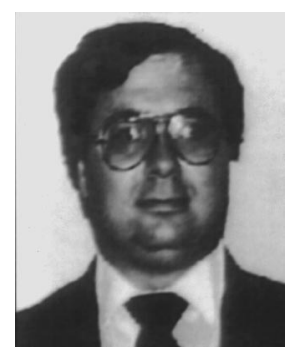

Arthur R. Weeks received his $\mathrm{PhD}$ in electrical engineering from the University if Central Florida in Orlando in 1987. He is an assistant professor of electrical and computer engineering at the University of Central Florida. His interests include the reduction of noise within images using adaptive nonlinear filters, color image processing, and the use of artificial neural networks in pattern recognition. Dr. Weeks is an associate editor of the Journal of Electronic Imaging and is a member of the IEEE, SPIE, and Tau Beta Pi. 